\title{
Genetic and diet effects on Ppar- $\alpha$ and Ppar- $\gamma$ signaling pathways in the Berlin Fat Mouse Inbred line with genetic predisposition for obesity
}

Asja Wagener ${ }^{1}$, Helge F Goessling ${ }^{1}$, Armin O Schmitt ${ }^{1}$, Susanne Mauel ${ }^{2}$, Achim D Gruber ${ }^{2}$, Richard Reinhardt ${ }^{3}$, Gudrun A Brockmann ${ }^{1 *}$

\begin{abstract}
Background: The Berlin Fat Mouse Inbred (BFMI) line is a new mouse model for obesity, which was long-term selected for high fatness. Peroxisome proliferator-activated receptors (PPARs) are involved in the control of energy homeostasis, nutrient metabolism and cell proliferation. Here, we studied the expression patterns of the different Ppar genes and the genes in the PPAR pathway in the BFMl line in comparison to physiological changes.

Results: At the age of 10 weeks, the BFMI mice exhibited marked obesity with enlarged adipocytes and high serum triglycerides concentrations in comparison to the often used mouse line C57BL/6 (B6). Between these two lines, gene expression analyses revealed differentially expressed genes belonging to the PPAR pathway, in particular genes of the lipogenesis and the fatty acid transport.

Conclusion: Surprisingly, the Ppar- $\alpha$ gene expression was up-regulated in liver and Ppar- $\gamma$ gene expression was down-regulated in the white adipose tissue, indicating the activation of a mechanism that counteracts the rise of obesity.
\end{abstract}

\section{Background}

The peroxisome proliferator-activated receptors (PPARs) constitute a family of three genes, which are involved in the control of energy homeostasis and cell proliferation. The PPAR family members have distinct patterns of tissue distribution and tissue specific functions. PPAR- $\alpha$ is predominantly present in liver where it has a critical role in the regulation of nutrient metabolism as it stimulates the uptake and oxidation of fatty acids. PPAR- $\gamma$ is mainly expressed in adipose tissue. It is induced during adipocyte differentiation and is a regulator in the formation of fat cells and lipid accumulation. PPAR- $\delta$ is abundantly expressed throughout the body and it has been proposed to be involved in adipogenesis and energy metabolism [for review, see [1-3]]. Activation of PPAR- $\alpha$ by agonists leads to reduced adiposity and

\footnotetext{
* Correspondence: gudrun.brockmann@agrar.hu-berlin.de

${ }^{1}$ Humboldt-Universität zu Berlin, Department for Crop and Animal Sciences, Invalidenstraße 42, 10115 Berlin, Germany

Full list of author information is available at the end of the article
}

lowered triglyceride levels by reduced food intake [4-6], whereas activation of PPAR- $\gamma$ by agonist stimulates lipid storage and is associated with body weight gain $[7,8]$.

Recently, we have generated the high-fatness selected Berlin Fat Mouse inbred line BFMI as a model for juvenile obesity [9]. BFMI mice harbour natural mutations leading to a five fold increased fat percentage due to hyperphagia at young age and an altered lipid metabolism in comparison to C57BL/6 mice [10,11]. A specific regulation of PPAR genes in the development of obesity in the BFMI mice is very likely.

Therefore, the intention of this study was to investigate whether the Ppars and their responsive genes are involved in the fat accumulation of the BFMI line. Our aims were (i) to identify differences in gene regulation of Ppars and their responsive genes between the obese BFMI mouse model and B6 mice as a control and (ii) to analyze responses of PPAR pathway genes to high-fat diet feeding in BFMI mice. Therefore, we analysed transcript amounts of genes belonging to the PPAR pathway 
in white adipose tissue and liver. Furthermore, we analysed body composition, adipocyte size and serum parameters.

\section{Results}

Phenotypic differences between lines BFMI and B6

To compare the body composition of the two lines, male mice of BFMI and B6 were fed a standard maintenance diet (SMD) until week 10. On SMD, animals of the BFMI line were significantly heavier and had more body fat and lean mass than B6 mice over the entire period (Figure 1). At 10 weeks, mice of the BFMI line were 1.8 times as heavy as B6 mice $(41.4 \pm 2.9 \mathrm{~g}$ and $23.5 \pm 1.5 \mathrm{~g}$, respectively). The body weight gain was due to increased fat mass. BFMI mice had almost 10 times as much body fat mass but only 1.5 times as much lean mass as B6 animals. In BFMI mice, fat was accumulated in all three investigated adipose tissues mainly due to enlarged adipocytes (Table 1, Figure 2 and 3). The weight of the liver was slightly higher in 10 weeks old BFMI mice compared to B6 mice which could be due to increased fat accumulation in liver of BFMI mice (Table 1, Figure 3). Serum triglyceride concentrations, but not total cholesterol concentrations, were elevated in BFMI mice compared to B6.

Feeding of HFD in mice of the BFMI line caused an additional body weight and body fat mass gain $(9.0 \mathrm{~g}$ and $6.4 \mathrm{~g}$, respectively). The excess amount of white adipose tissue (WAT) was more pronounced in subcutaneous and renal adipose tissue than in the reproductive adipose tissues (Table 1). This is in accordance with the observation that subcutaneous white adipose tissue is known as a primary fat storage depot. However, adipocytes were only slightly increased in subcutaneous adipose and not further increased in reproductive and renal adipose tissue indicating that proliferation of adipocytes occurs to uptake the additional fat in HFD-fed BMFI mice (Figure 2). Liver weight was significantly increased in mice on HFD due to increased fat accumulation (Figure 3). Serum triglyceride concentrations were not further increased in BFMI mice on HFD, but serum total cholesterol concentrations were elevated (Table 1).

\section{Comparison of genes belonging to the PPAR pathway between BFMI and B6 mice}

Among the members of the PPAR signalling pathway (KEGG database), 20 and 19 genes were differentially expressed between the lines BFMI and B6 on SMD in adipose tissue and in liver, respectively (Table 2, Figure 4).

On SMD, the transcript amount of Ppar- $\alpha$, Ppar- $\gamma$ and their receptors Rxra and $R x r g$ in reproductive adipose tissue were lower in BFMI mice compared to B6. Correspondingly, down-stream activated genes of the fatty
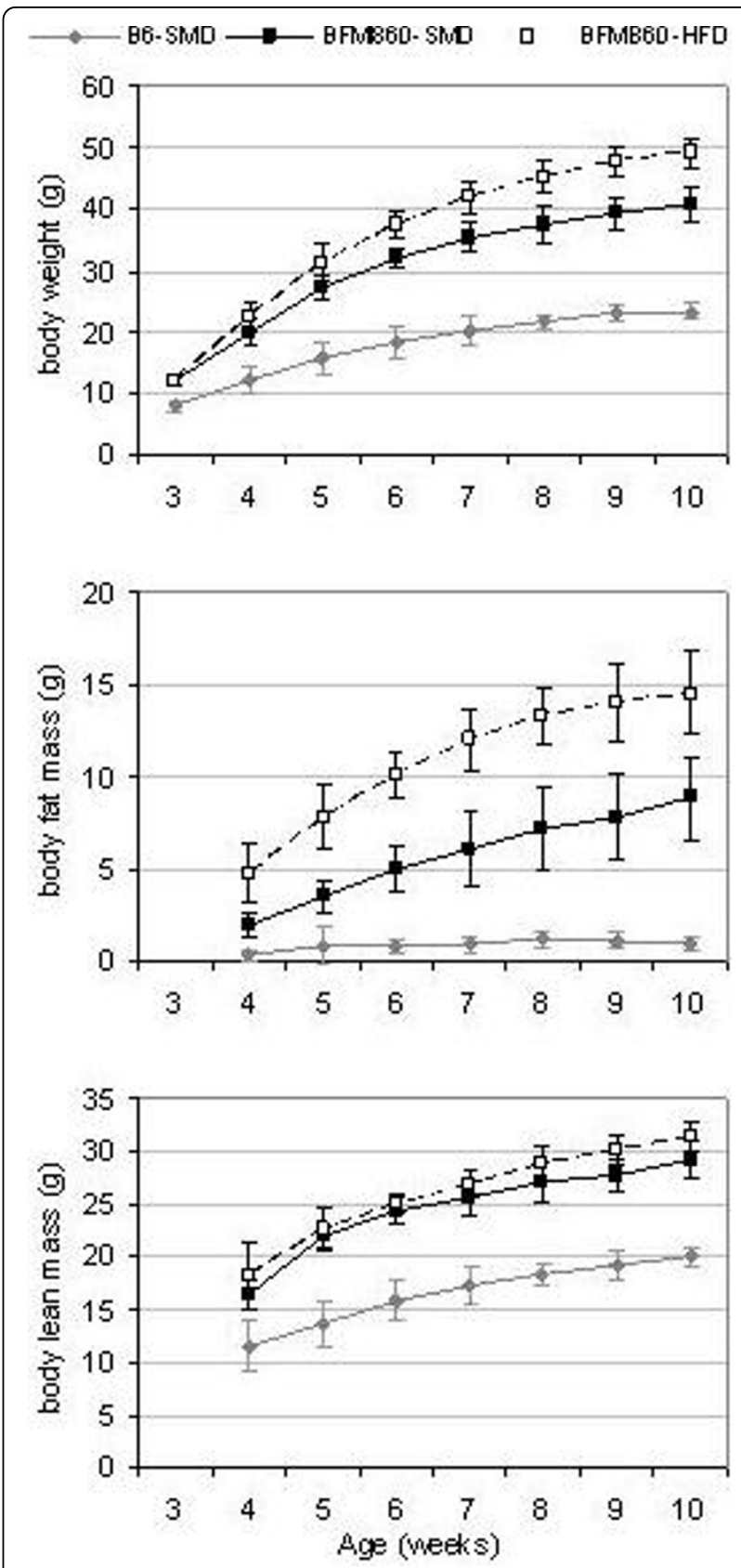

Figure 1 Comparisons of lines BFMI and B6 on standard maintenance diet (SMD) and of line BFMI on high-fat diet (HFD). Body weight, body fat mass and body lean mass from three to 10 weeks of age. Lines differ from 3 weeks of age on and animals between the two diet groups differ from 4 weeks of age in line BFMI $(P<0.05)$. Each point represents the mean weight with standard deviation.

acid oxidation but also genes of the lipogenesis were down-regulated. Within the fatty acid oxidation pathway, members of the cytochrome family, the acetyl-CoA acyltransferase (Acaa1) and the carnitine palmitoyltransferase 2 (Cpt2) were decreased. Lipogenesis in BFMI 
Table 1 Body composition and serum parameters in 10 week old BFMI and B6 mice on SMD and BFMI mice on HFD

\begin{tabular}{lccc}
\hline Trait & B6 & $\begin{array}{c}\text { BFMI on } \\
\text { SMD }\end{array}$ & $\begin{array}{c}\text { BFMI on } \\
\text { HFD }\end{array}$ \\
\hline Body weight (g) & $23.4(1.4)$ & $39.2(2.7)^{*}$ & $47.8(2.3)+$
\end{tabular}

Body composition traits

$\begin{array}{lccc}\text { Body lean mass (g) } & \begin{array}{c}19.97 \\ (0.95)\end{array} & 29.29(1.71)^{*} & 31.43(1.38)+ \\ \text { Body fat mass (g) } & 0.97(0.31) & 8.84(2.26)^{*} & 14.56(2.26)+ \\ \text { Total white fat tissue (g) } & 1.11(0.26) & 7.70(1.70)^{*} & 12.10(0.95)+ \\ \text { Reproductive fat pad (g) } & 0.23(0.05) & 1.96(0.43)^{*} & 2.26(0.53) \\ \text { Renal fat pad (g) } & 0.07(0.03) & 0.69(0.13)^{*} & 1.01(0.19)+ \\ \text { Subcutaneous fat pad (g) } & 0.50(0.15) & 2.57(1.02)^{*} & 4.42(1.42)+ \\ \text { Liver (g) } & 1.16(0.15) & 2.19(0.22)^{*} & 3.33(0.46)+\end{array}$

Serum parameter traits

Triglycerides (mg/dl) $123(63) \quad 277(95)^{*} \quad 245(97)$

Total cholesterol (mg/dl) $103(20) \quad 100(11) \quad 177(24)+$

SMD, standard maintenance diet; HFD, high-fat diet; values represent mean values with standard deviation in parenthesis of B6 on SMD $(n=15)$, BFMI on $\operatorname{SMD}(n=11)$, and BFMI on HFD $(n=12)$. Serum parameters were obtained after a fasting period of two hours (BFMI on SMD $(n=8)$, BFMI on HFD $(n=8)$, and B6 $(n=12))$. Asterisks indicate significant differences between BFMI860 and B6. Except for cholesterol all values were significantly different between B6 and BFMI mice on SMD. + indicates significant differences between standard maintenance and high-fat diet within line BFMI860 $(\mathrm{P}<0.05)$.

mice was characterized by down-regulation of the malic enzyme (Mod1), which provides energy rich molecules for the fatty acid synthesis.

Transcript amounts of genes encoding phospholipid transfer protein (Pltp), Cd36 antigen, fatty acid binding protein (Fabp3) and Acyl-CoA-synthetase (Acsl4), which are responsible for lipid and fatty acid transport, were upregulated in BFMI mice compared to B6. The uncoupling protein $1(U c p 1)$ that enhances thermogenesis was also

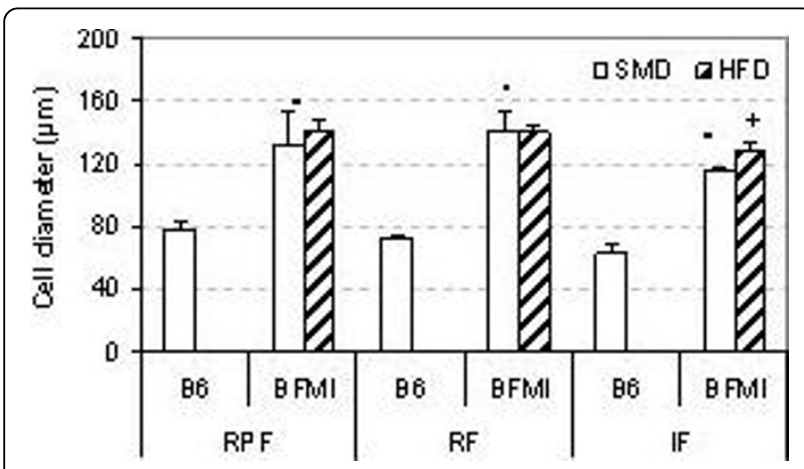

Figure 2 Adipocyte diameter of reproductive (RPF), retroperitoneal (RF), and inguinal (IF) adipose tissue of $B 6$ and BFMI mice on standard maintenance diet (SMD) and of BFMI mice on high-fat diet (HFD). Animals were 10 weeks old. highly up-regulated in reproductive adipose tissue in BFMI mice.

Unlike in adipose tissue, the expression of Ppar- $\alpha$ and its receptors Rxra and Rxrg were increased in liver. As in adipose tissue, genes of the lipid or fatty acid transport (here Apoa2, Apoa5 and Fabp3) were more expressed in liver of BFMI mice than in B6 mice. Genes involved in fatty acid oxidation were differentially expressed between both mouse lines. Liver transcript amounts of Acyl-CoA oxidase (Acox2) and acyl-Coenzyme A dehydrogenase $(A c a d m)$ were higher, whereas 3-hydroxyacyl Coenzyme A dehydrogenase (Ehhadh), Cpt 2 and members of the cytochrome P450 family were lower in BFMI than in B6.

\section{Diet effect on genes of the PPAR pathway in BFMI mice} To determine whether dietary fat regulates the expression of genes of the PPAR pathway in BFMI mice, mRNA expression profiles of reproductive adipose tissue and liver of mice on SMD and HFD were compared. In response to HFD, the mRNA expression of Ppar- $\gamma$ and its receptor was down-regulated in reproductive adipose tissue, whereas the expression of Ppar- $\alpha$ and its receptor were reduced in liver. Transcriptional response in both tissues indicated that most genes involved in lipid metabolism were up-regulated (Table 2, Figure 4). An exception was the cholesterol metabolism, which was reduced. In particular, genes involved in lipogenesis (Scd1, Mod1) but also in peroxisomal and mitochondrial fatty acid oxidation (Acaa1, Cpt2) and in ketogenesis (Hmgcs2) were up-regulated in response to HFD.

In addition, the expression of genes of the fatty acid transport (Acsl4, Dbi, Fabp3) and of the cell survival $(P d p k 1)$ were increased in reproductive adipose tissue. The Ucp1 expression was further elevated in line BFMI. Genes affecting adipocyte differentiation, like angiopoietin-like 4 (Angptl4), which is involved in the cellular response to starvation, and adiponectin were downregulated in this tissue in response to HFD-feeding. In liver tissue, genes belonging to lipid transport (Apoa1, Apoa3) were further up-regulated.

\section{Discussion}

The BFMI line is a model for studies of obesity and subsequent complications, which are close to obese humans. The purpose of the presented work was to evaluate the role of different Ppar genes and their responsive genes in the fat accumulation in the BFMI mice as PPARs are important regulators of lipid metabolism and energy homeostasis. Increased fat accumulation accompanied with increased adipocyte size and elevated serum triglyceride levels, observed in BFMI compared to B6 mice in this study, makes a change of Ppar gene expression and their responsive genes very likely. As PPAR- $\alpha$ agonists lead to 


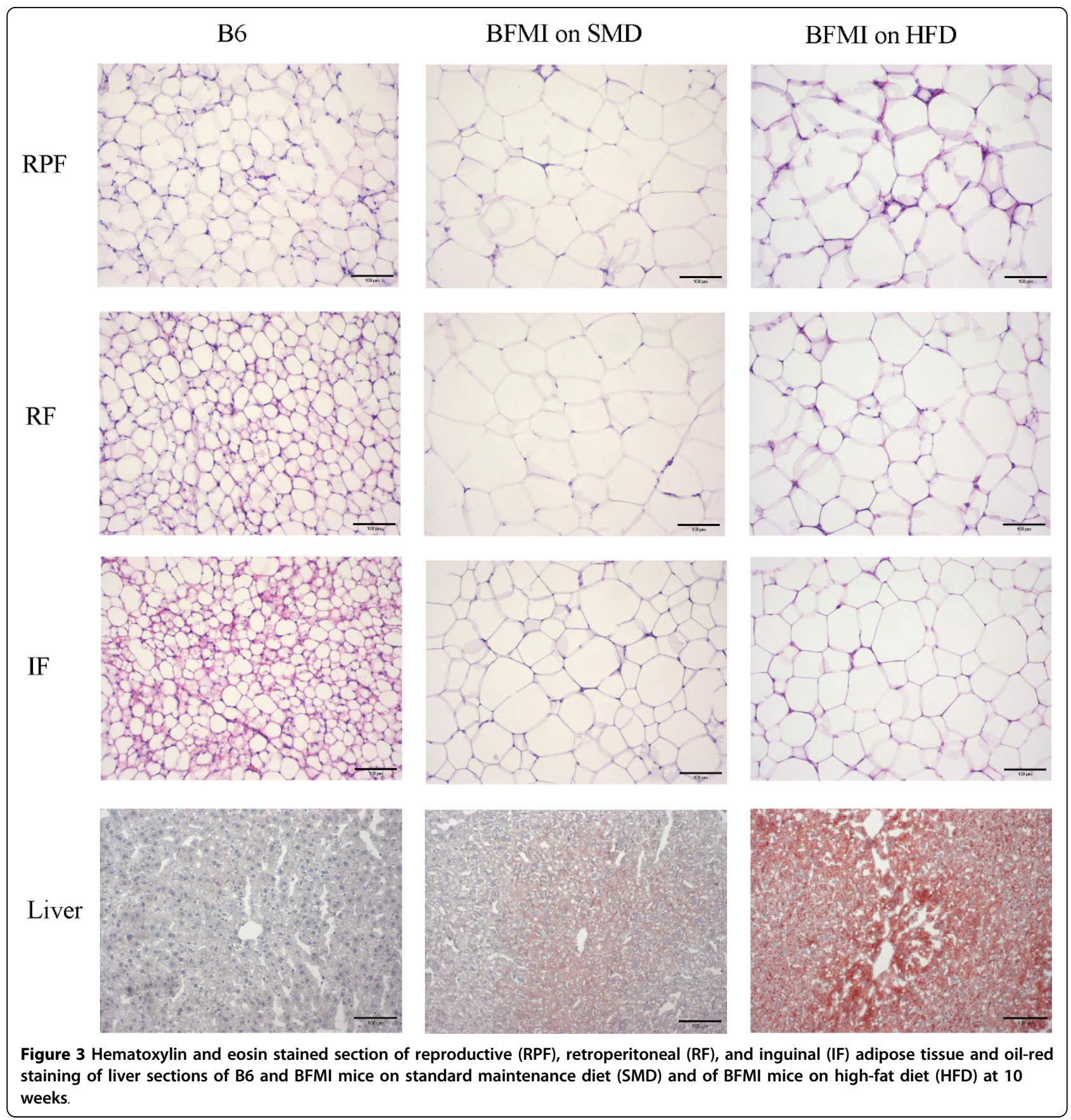

reduced adiposity $[4,5]$ and PPAR- $\gamma$ agonists stimulate lipid storage and body weight gain $[7,8]$ we would have expected a down-regulation of Ppar- $\alpha$ in liver and an upregulation of Ppar- $\gamma$ in adipose tissue in BFMI mice compared to B6. In contrast to our expectation, transcript amounts in white adipose tissue and in liver revealed a down-regulation of Ppar- $\alpha$ and Ppar- $\gamma$ and their receptors in adipose tissue and an up-regulation of Ppar- $\alpha$ and its receptor in liver in the high-fatness selected BFMI line compared to B6. In response to HFD, BFMI mice showed a further reduction of Ppar- $\gamma$ and Rxrg mRNA levels in adipose tissue but a reduced mRNA level of Ppar- $\alpha$ and its receptor in liver.

The observation that Ppar- $\alpha$ mRNA levels are increased in liver of BFMI mice compared to B6 corresponds well with findings in $o b / o b$ and $d b / d b$ mice in comparison with lean mice [12]. But this is in contrast with findings in Ppar- $\alpha$ deficient mice which had higher fat mass, liver weight and triglyceride concentrations $[13,14]$. In obese mice, PPAR- $\alpha$ might be activated to 
Table 2 Changes in expression of genes involved in the PPAR pathway.

\begin{tabular}{|c|c|c|c|c|c|c|c|c|c|}
\hline \multirow{3}{*}{ Entrez Gene-IDs } & \multirow[t]{3}{*}{ Gene symbol } & \multicolumn{4}{|c|}{ BFMI860/B6 (SMD) } & \multicolumn{4}{|c|}{ HFD/SMD (BFMI860) } \\
\hline & & \multicolumn{2}{|c|}{ Fat } & \multicolumn{2}{|c|}{ liver } & \multicolumn{2}{|c|}{ fat } & \multicolumn{2}{|c|}{ liver } \\
\hline & & Fold ch. & p-value & Fold ch. & p-value & Fold ch. & p-value & Fold ch. & $p$-value \\
\hline \multicolumn{10}{|c|}{ PPAR isoforms and receptors } \\
\hline 19013 & Ppara & 0.87 & $<0.01$ & 2.67 & $<0.01$ & 0.95 & ns & 0.48 & $<0.01$ \\
\hline 19015 & Ppard & 1.07 & ns & 0.98 & ns & 0.73 & ns & 0.88 & ns \\
\hline 19016 & Pparg & 0.66 & $<0.01$ & 0.83 & $<0.05$ & 0.70 & $<0.01$ & 0.96 & ns \\
\hline 20181 & Rxra & 0.69 & $<0.01$ & 1.09 & $<0.05$ & 0.95 & ns & 0.89 & $<0.05$ \\
\hline 20182 & Rxrb & 0.98 & ns & 1.06 & ns & 1.00 & ns & 1.00 & ns \\
\hline 20183 & Rxrg & 0.84 & $<0.01$ & 1.29 & $<0.01$ & 0.81 & $<0.01$ & 1.02 & ns \\
\hline \multicolumn{10}{|l|}{ Lipid transport } \\
\hline 11806 & Apoa1 & 0.97 & ns & 0.96 & ns & 1.04 & ns & 1.11 & $<0.01$ \\
\hline 11807 & Apoa2 & 0.97 & ns & 1.21 & $<0.05$ & 1.16 & ns & 1.02 & ns \\
\hline 66113 & Apoa5 & 0.94 & ns & 1.32 & $<0.05$ & 1.00 & ns & 0.92 & ns \\
\hline 11814 & Apoc3 & 0.98 & ns & 0.94 & ns & 0.98 & ns & 1.15 & $<0.01$ \\
\hline 18830 & PLTP & 2.32 & $<0.01$ & 0.75 & ns & 0.64 & ns & 1.26 & ns \\
\hline \multicolumn{10}{|l|}{ Fatty acid transport } \\
\hline 14081 & Acsl1 & 0.97 & ns & 0.97 & ns & 0.82 & ns & 0.84 & ns \\
\hline 74205 & Acsl3 & 1.03 & ns & 1.01 & ns & 0.98 & ns & 0.94 & ns \\
\hline 50790 & Acsl4 & 1.14 & $<0.05$ & 0.98 & ns & 1.18 & $<0.05$ & 1.03 & ns \\
\hline 433256 & Acsl5 & 1.09 & ns & 1.02 & ns & 1.05 & ns & 1.12 & ns \\
\hline 216739 & Acsl6 & 0.97 & ns & 0.99 & ns & 1.01 & ns & 1.06 & ns \\
\hline 12491 & $\mathrm{Cd} 36$ & 1.61 & $<0.01$ & 1.06 & ns & 1.16 & ns & 1.42 & $<0.05$ \\
\hline 13167 & Dbi & 0.86 & ns & 0.92 & ns & 1.39 & $<0.01$ & 1.08 & ns \\
\hline 14080 & Fabp1 & 1.09 & ns & 1.04 & ns & 0.97 & ns & 0.87 & $<0.05$ \\
\hline 14079 & Fabp2 & 1.05 & ns & 1.08 & ns & 0.95 & ns & 0.85 & $<0.01$ \\
\hline 14077 & Fabp3 & 1.36 & $<0.05$ & 1.15 & $<0.05$ & 1.83 & $<0.01$ & 0.90 & ns \\
\hline 11770 & Fabp4 & 1.00 & ns & 1.01 & ns & 1.26 & ns & 0.95 & ns \\
\hline 16592 & Fabp5 & 1.47 & ns & 0.88 & ns & 1.38 & ns & 0.99 & ns \\
\hline 16204 & Fabp6 & 0.94 & $<0.01$ & 1.02 & ns & 1.02 & ns & 1.04 & ns \\
\hline 12140 & Fabp7 & 1.07 & ns & 0.96 & ns & 0.97 & ns & 0.96 & ns \\
\hline 16956 & Lpl & 0.85 & $<0.01$ & 1.02 & ns & 1.04 & ns & 0.95 & ns \\
\hline 108078 & Olr1 & 0.96 & ns & 1.01 & ns & 0.99 & ns & 0.95 & ns \\
\hline 26457 & Slc27a1 & 0.75 & ns & 0.97 & ns & 0.99 & ns & 1.03 & ns \\
\hline 26458 & Slc27a2 & 1.02 & ns & 0.97 & ns & 0.97 & ns & 1.15 & ns \\
\hline 26569 & Slc27a4 & 1.07 & ns & 1.01 & ns & 0.92 & $<0.05$ & 0.99 & ns \\
\hline 26459 & Slc27a5 & 0.99 & ns & 1.06 & ns & 1.00 & ns & 0.83 & $<0.01$ \\
\hline 225579 & Slc27a6 & 1.14 & $<0.01$ & 0.82 & $<0.01$ & 0.96 & ns & 1.11 & $<0.05$ \\
\hline \multicolumn{10}{|l|}{ Fatty acid oxidation } \\
\hline 113868 & Acaa1a & 0.78 & $<0.05$ & 0.97 & ns & 1.46 & $<0.05$ & 1.15 & $<0.05$ \\
\hline 11363 & Acadl & 0.91 & ns & 1.1 & ns & 1.09 & ns & 1.18 & ns \\
\hline 11364 & Acadm & 0.7 & ns & 1.89 & $<0.01$ & 0.93 & ns & 1.08 & ns \\
\hline 11430 & Acox 1 & 1.07 & ns & 1.74 & ns & 1.06 & ns & 0.67 & ns \\
\hline 93732 & Acox 2 & 1.05 & ns & 1.76 & $<0.01$ & 0.97 & ns & 0.80 & ns \\
\hline 80911 & Acox3 & 0.99 & ns & 1.01 & ns & 0.99 & ns & 0.99 & ns \\
\hline 12894 & Cpt1a & 0.98 & ns & 1.01 & ns & 0.99 & ns & 0.94 & ns \\
\hline 12895 & Cpt1b & 1.17 & ns & 0.99 & ns & 1.20 & $<0.05$ & 1.07 & ns \\
\hline 78070 & Cpt1c & 0.96 & ns & 1.01 & ns & 0.97 & ns & 0.98 & $<0.05$ \\
\hline 12896 & Cpt2 & 0.71 & $<0.01$ & 0.63 & $<0.01$ & 1.61 & $<0.05$ & 1.85 & $<0.01$ \\
\hline 13117 & Cyp4A10 & 1.00 & ns & 0.24 & $<0.01$ & 0.98 & ns & 5.07 & $<0.01$ \\
\hline 13118 & Cyp4a12b & 1.03 & ns & 0.80 & ns & 0.94 & ns & 0.59 & $<0.05$ \\
\hline 13119 & Cyp4a14 & 0.90 & $<0.01$ & 0.06 & $<0.01$ & 1.06 & ns & 31.29 & $<0.01$ \\
\hline 74147 & Ehhadh & 0.98 & ns & 0.69 & $<0.05$ & 0.89 & ns & 2.17 & $<0.01$ \\
\hline 20280 & Scp2 & 0.85 & ns & 0.95 & ns & 0.87 & ns & 0.91 & ns \\
\hline
\end{tabular}


Table 2: Changes in expression of genes involved in the PPAR pathway. (Continued)

\begin{tabular}{|c|c|c|c|c|c|c|c|c|c|}
\hline \multicolumn{10}{|l|}{ Ketogenesis } \\
\hline 15360 & Hmgcs2 & 0.92 & ns & 1.27 & ns & 1.72 & $<0.01$ & 1.14 & $<0.05$ \\
\hline \multicolumn{10}{|l|}{ Lipogenesis } \\
\hline 56473 & Fads2 & 1.05 & ns & 0.69 & ns & 0.93 & ns & 3.16 & $<0.01$ \\
\hline 677317 & Mod1 & 0.58 & $<0.01$ & 0.75 & ns & 2.26 & $<0.01$ & 2.81 & $<0.01$ \\
\hline 20249 & Scd1 & 0.76 & $<0.01$ & 0.81 & $<0.05$ & 1.45 & $<0.01$ & 1.33 & $<0.01$ \\
\hline 20250 & $\mathrm{Scd} 2$ & 1.26 & ns & 1.46 & $<0.01$ & 2.03 & $<0.01$ & 1.16 & ns \\
\hline 30049 & Scd3 & 1.03 & ns & 0.98 & ns & 0.98 & ns & 1.02 & ns \\
\hline 329065 & $\mathrm{Scd} 4$ & 1.01 & ns & 1.06 & ns & 0.98 & ns & 1.00 & ns \\
\hline \multicolumn{10}{|c|}{ Cholesterol metabolism } \\
\hline 104086 & Cyp27a1 & 1.04 & ns & 1.00 & ns & 0.78 & $<0.01$ & 0.70 & $<0.01$ \\
\hline 13122 & Cyp7a1 & 0.96 & ns & 2.31 & ns & 1.00 & ns & 0.49 & ns \\
\hline 13124 & Cyp8b1 & 1.00 & ns & 1.37 & $<0.05$ & 0.99 & ns & 1.04 & ns \\
\hline 22259 & Nr1h3 & 0.96 & ns & 0.92 & ns & 0.76 & $<0.05$ & 1.11 & ns \\
\hline \multicolumn{10}{|c|}{ Gluconeogenesis } \\
\hline 11832 & Aqp7 & 0.83 & ns & 1.04 & ns & 1.12 & ns & 1.03 & ns \\
\hline 14626 & Gk2 & 1.02 & ns & 1.01 & ns & 1.05 & ns & 1.03 & ns \\
\hline 14933 & Gyk & 1.01 & ns & 0.96 & ns & 1.00 & ns & 1.00 & ns \\
\hline 18534 & Pck1 & 0.65 & ns & 0.54 & ns & 0.87 & ns & 1.15 & ns \\
\hline 74551 & Pck2 & 0.99 & ns & 1.17 & $<0.01$ & 0.97 & ns & 0.86 & $<0.05$ \\
\hline \multicolumn{10}{|c|}{ Adipocyte differentiation } \\
\hline 11450 & Adipoq & 0.71 & $<0.05$ & 0.97 & ns & 0.49 & $<0.01$ & 1.01 & ns \\
\hline 57875 & Angpt|4 & 1.41 & ns & 0.84 & ns & 0.71 & $<0.01$ & 1.04 & ns \\
\hline 83995 & Mmp1a & 1.02 & ns & 0.99 & ns & 0.99 & ns & 1.00 & ns \\
\hline 83996 & Mmp1b & 1.06 & ns & 0.97 & ns & 0.97 & ns & 1.04 & ns \\
\hline 103968 & Plin & 0.70 & ns & 0.98 & ns & 0.68 & ns & 1.03 & ns \\
\hline 20411 & Sorbs1 & 0.95 & ns & 0.94 & ns & 1.04 & ns & 1.06 & ns \\
\hline \multicolumn{10}{|c|}{ Adaptive thermogenesis } \\
\hline 22227 & Ucp1 & 2.65 & $<0.01$ & 1.03 & ns & 2.87 & $<0.01$ & 0.98 & ns \\
\hline \multicolumn{10}{|l|}{ Cell survival } \\
\hline 16202 & Ilk & 1.27 & ns & 0.83 & ns & 0.77 & ns & 1.14 & $<0.05$ \\
\hline 18607 & Pdpk1 & 0.62 & $<0.01$ & 1.38 & $<0.01$ & 1.61 & $<0.05$ & 0.83 & $<0.05$ \\
\hline \multicolumn{10}{|c|}{ Ubiquitination } \\
\hline 22190 & Ubc & 0.87 & ns & 1.25 & ns & 0.83 & ns & 0.76 & ns \\
\hline
\end{tabular}

Changes in expression are expressed as fold changes in BFMI860 in comparison to B6 mice on SMD, and of HFD-fed BFMI860 mice compared to SMD-fed mice, respectively. SMD- standard maintenance diet, HFD- high-fat diet, ns-not significant

counteract the obese state. In BFMI mice, PPAR responsive genes involved in lipid transport are up-regulated, but despite the up-regulation of PPAR- $\alpha$ expression genes involved in fatty acid oxidation are down-regulated, which leads to fat accumulation in the liver. The accumulation of fat in the liver suggests preferred usage of glucose for oxidation, which is consistent with higher respiratory quotients in BFMI mice [10].

BFMI mice responded to HFD by a reduction of PPAR- $\alpha$ expression in liver. This is in line with increased adiposity in Ppar- $\alpha$ null mice after HFD-feeding [4]. Our finding is in contrast with the increased PPAR- $\alpha$ expression in diet-induced obese B6 mice, in which it goes along with elevated fatty acid oxidation [14]. In BFMI mice, genes of lipid transport, lipogenesis, fatty acid oxidation and ketogenesis were up-regulated despite the reduced Ppar expression. This finding suggests hat the PPAR- $\alpha$ signalling in BFMI mice might be disturbed or that other factors have a stronger influence on lipid metabolism. Despite elevated fatty acid utilization in the liver the high lipid storage resulted in a fatty liver in HFD-fed BFMI mice.

PPAR- $\gamma$ activation is essential for the formation of adipocytes and, as a consequence, Ppar- $\gamma$ knockout mice fail to develop adipose tissue [7,8]. In $d b / d b$ and $o b / o b$ mice PPAR- $\gamma$ agonism or activation led to the reduction of serum concentrations of triglycerides [5,15]. Surprisingly, in BFMI mice Ppar- $\gamma$ is down-regulated despite high body fat mass and increased adipocyte size in comparison to B6. In the white adipose tissue, the decreased 


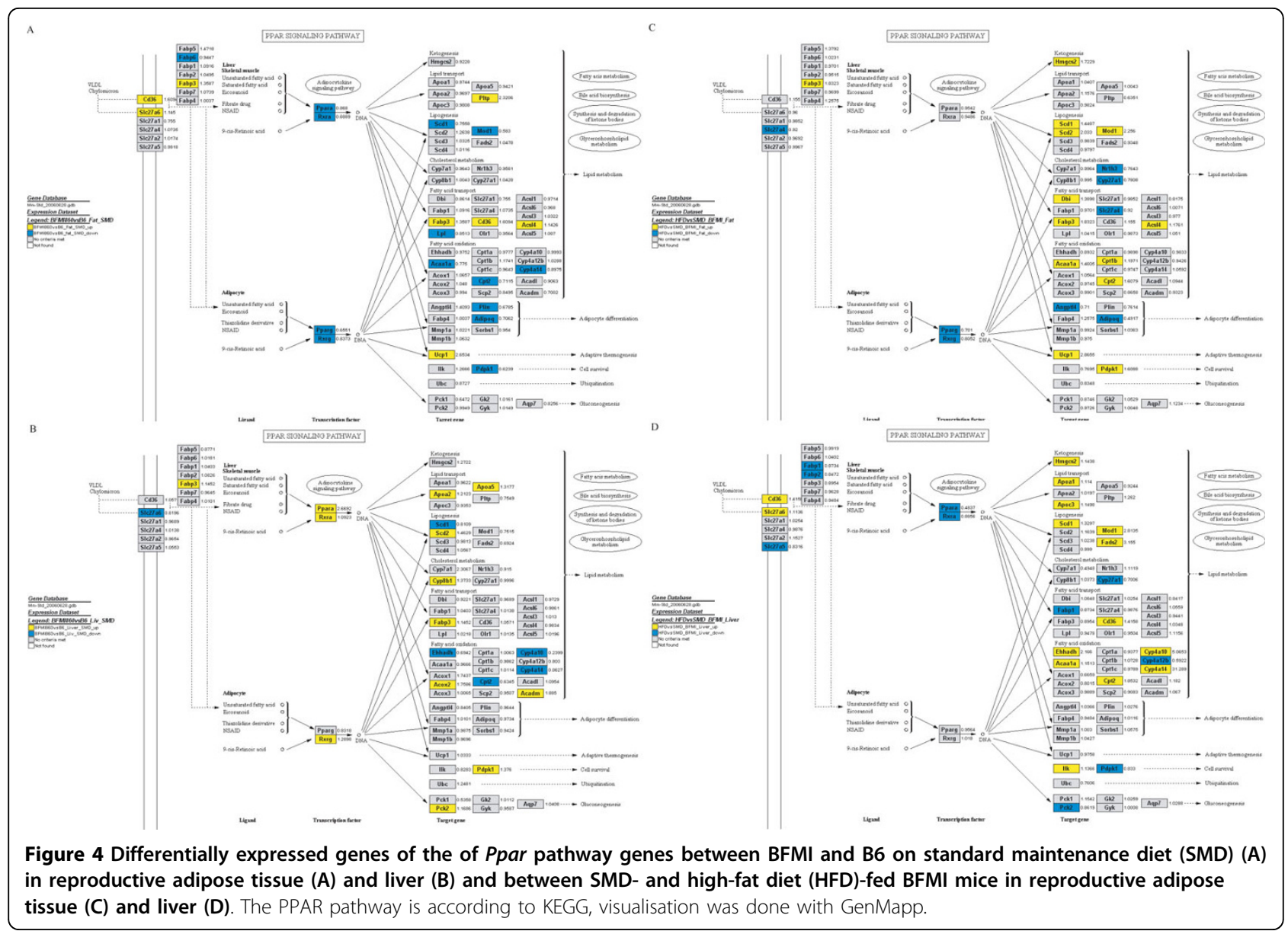

Ppar- $\gamma$ expression in BFMI mice goes along with decreased fatty acid oxidation. This coincides with the observation in Zucker rats, in which PPAR- $\gamma$ agonism led to stimulation of genes involved in fatty acid oxidation [16]. In BFMI mice, reduction of fatty acid oxidation might be also due to the interaction with Ppar- $\alpha$, which is also lowered in adipose tissue. Reduced fatty acid oxidation and elevated fatty acid transport in BFMI mice compared to B6 lead to an elevated fat deposition and increased white adipose tissues with hypertrophic adipocytes in BFMI mice. As the proportion of the adipose tissue weights between BFMI and B6 mice was larger than the proportion of the adipocyte size between the lines, we suggest that proliferation of adipocytes still occurs in BFMI mice.

However, there were also fat storage counteracting effects in BFMI mice. The expression of Ucp1, which facilitates thermogenesis, was highly increased in line BFMI in comparison to B6. UCPs could dispose excess energy by uncoupling mitochondrial respiration from oxidative phosphorylation under fatty acid consumption [17]. In response to HFD, Ucp 1 as well as genes of the fatty acid oxidation (Acaa1a, Cpt1b, Cpt2) were further elevated in adipose tissue of BFMI mice. Moreover, in adipose tissue and liver of BFMI mice on HFD, fatty acids seem to be converted to ketones as energy sources. The high amount of $U_{c p} 1$ transcripts and the change of fatty acid utilisation indicate a counteraction to the excessive energy supply and might explain the unchanged triglyceride levels in BFMI mice on SMD and HFD. An up-regulation of $U c p 1$ in response to HFD was also seen in DBA/2J mice [18]. The increase in fat mass in HFD-fed mice mirrors further lipid storage as a result of fatty acid uptake and lipogenesis which were up-regulated. As the adipocyte size was not further increased, but tissue mass nearly doubled in HFD-fed BFMI mice compared to SMD, cell proliferation still takes place, thus developing additional adipocytes. This is indicated by an up-regulation of $P d p k 1$, which is involved in cell survival. Surprisingly, new adipocytes are developed in BFMI mice despite the decreased Ppar- $\gamma$ expression in response to HFD. This is in contrast with findings in heterozygous Ppar- $\gamma$ mutant mice that had smaller fat pads [19]. In BFMI mice, down-regulation of Ppar- $\gamma$ expression seems to counteract fat accumulation rather than to promote fat storage. 
PPAR- $\delta$ is abundantly expressed throughout the body, but little is known about its role in lipid metabolism [2]. Although it seems to play a role in obesity [20], Ppar- $\delta$ expressions of reproductive adipose tissue and liver were similar in BFMI and B6 mice. Furthermore, they were not changed by the diet. The same was found in liver of $o b / o b$ and $d b / d b$ mice in comparison to B6 [12].

In summary, elevated fatty acid and lipid transport into adipose tissue accompanied by lower fatty acid oxidation contribute to hypertrophy of adipocytes and excess of body fat mass in the high-fatness selected BFMI line. Our results suggest that the regulation of Ppar genes in BFMI mice is a strategy of the organism to counteract the rise of obesity rather than promoting the formation of the obese phenotype. However, the signalling from Ppar genes to the fatty acid oxidation seems to be disturbed. In response to HFD, likewise mechanisms seem to be activated that hamper fat gain. In HFD-fed BFMI mice, the fatty acid oxidation, ketogenesis and thermogenesis are up-regulated indicating increased utilization of fatty acids as energy source. Despite increased fatty acid oxidations, elevated lipogenesis led to additional fat stored in the adipose tissue and fatty liver in HFD-fed BFMI mice.

Considering the many differences in PPAR gene expression in comparison to $\mathrm{B} 6$ mice and in the response to HFD, BFMI mice provide a new model for the study of PPAR actions under controlled genetic and environmental conditions.

\section{Methods}

\section{Animals and diets}

In this study, we used males of the BFMI860 line which was generated from an outbred population. Founders of the BFMI line were originally purchased from pet shops and were consecutively selected for low protein content, low body mass and high fat content, and for high fatness for 58 generations before inbreeding [9]. C57BL/ 6NCrl (B6) mice (Charles River Laboratories, Sulzfeld, Germany) were used as a control because this mouse line was a parental line for a cross-bred experiment to map genetic loci affecting body fat content in BFMI mice [11]. Mice were kept at room temperature $\left(22^{\circ} \mathrm{C}\right.$ $24^{\circ} \mathrm{C}$ ) with a light dark cycle of 12 hours. After weaning at the age of three weeks, 11 mice of the BFMI line and 15 mice of the B6 line were randomly chosen and fed a standard maintenance diet (SMD) containing $12.8 \mathrm{MJ} /$ $\mathrm{kg}$ metabolizable energy with $9 \%$ of its energy from fat, $33 \%$ from protein content and 58\% from carbohydrates (V1534-000 ssniff R/M-H, ssniff Spezialdiäten GmbH, Soest, Germany). Twelve additional males of the BFMI line were set on a high-fat diet (HFD) containing 19.1 $\mathrm{MJ} / \mathrm{kg}$ metabolizable energy with $45 \%$ of its energy from fat, $24 \%$ from protein content and $31 \%$ from carbohydrates (S8074-E010 ssniff EF R/M, ssniff Spezialdiäten $\mathrm{GmbH}$, Soest, Germany). The ingredients of both diets are given in Table 3 . The standard diet derived its fat from soy oil, whereas the high-fat diet derived its fat from coconut oil and suet. Animals had ad libitum access to water and diets. All animal treatments were in accordance with the German Animal Welfare Legislation (approval no. G0152/04).

\section{Phenotypes}

For the phenotypic characterization, animals were weighed weekly on the basis of their day of birth from the age of 3 weeks on. Body fat mass and body lean mass were also determined weekly in non-anesthetized animals by quantitative magnetic resonance (QMR) analysis using the EchoMRI whole body composition analyzer (Echo Medical Systems, Houston, Texas, USA) [21]. At 10 weeks, mice were fasted for two hours, anesthetized with isofluran and immediately killed by decapitation. After bleeding, different white adipose tissues (reproductive, renal, subcutaneous) and liver were dissected and weighed. The remaining white adipose tissues were collected and weighted. The sum of all visible white adipose tissues was termed 'total white fat tissue'.

\section{Histology}

For the histological examinations, the reproductive adipose tissue (around the testicles), the retroperitoneal (as part of the renal adipose tissue), the inguinal adipose tissue (as part of the subcutaneous adipose tissue) and the liver of three animals of each line and diet group were immediately fixed upon dissection in $4 \%$ buffered formaldehyde solution ( $\mathrm{pH}$ 7.4) for 24 hours. Adipose

Table 3 Ingredients of standard maintenance diet (SMD) and high-fat diet (HFD)

\begin{tabular}{lcc}
\hline Ingredient & SMD & HFD \\
\hline Crude nutrients & \multicolumn{2}{c}{ proportion [\%] } \\
Crude proteins & 19.0 & 20.7 \\
Crude fat & 3.3 & 25.0 \\
Crude fiber & 4.9 & 5.0 \\
Crude ashes & 6.7 & 5.9 \\
Dry substance & 87.7 & 96.3 \\
N-free extracts & 54.1 & 39.7 \\
Starch & 36.5 & 20.0 \\
Sugar & 4.7 & 17.5 \\
\hline Metabolizable energy & & MJ/kg \\
& 12.8 & 19.1 \\
Fat & & $\%$ of energy \\
Carbohydrate & 9.0 & 45.0 \\
Protein & 58.0 & 31.0 \\
\hline
\end{tabular}

Both diets contained also vitamins, trace elements, and minerals. 
tissue samples were embedded in paraffin following standard laboratory procedures. Sections $(3 \mu \mathrm{m})$ of dewaxed tissues were stained with hematoxylin and eosin. Adipocyte cell diameter was measured from 30 cells per tissue per mouse. Cells with a round shape were chosen for determination of the cell size. Cryosections $(10 \mu \mathrm{m})$ of liver tissue were incubated with $0.18 \%$ oil-red $\mathrm{O}$ for $10 \mathrm{~min}$, washed with $60 \%$ isopropanol, and counterstained with hematoxylin. Sections were photographed at 100x magnification with a digital camera.

\section{Serum parameters}

Blood was collected at slaughtering and serum was recovered by centrifugation for $15 \mathrm{~min}$ at $600 \mathrm{~g}$ and stored at $-20^{\circ} \mathrm{C}$ until analysis. Serum triglycerides and total cholesterol were determined using the Fluitest TG (Triglyceride GPO-PAP) and Fluitest Chol (Cholesterin CHOD-PAP) kits (both Biocon Medizintechnik, Mönchberg, Germany).

\section{RNA isolation and microarray analysis}

For gene expression analyses, the reproductive adipose tissue and liver of six mice per feeding group at ten weeks were immediately frozen in liquid nitrogen. We used the reproductive adipose tissue for gene expression analysis as visceral white adipose tissue is known to be more metabolically active than subcutaneous $[18,22]$. Total RNA was prepared using the NucleoSpin RNA II Kit (Macherey-Nagel, Düren, Germany) following the manufacturer's protocol. Quality of RNA was verified on an agarose gel with ethidium bromide staining. $400 \mathrm{ng}$ of RNA was reverse transcribed to synthesize first and second strand complementary DNA (cDNA), purified with spin columns and then in vitro transcribed to synthesize biotin-labelled complementary RNA (cRNA) (Ambion Illumina RNA Amplification Kit, Cambridgeshire, UK). $1.5 \mu \mathrm{g}$ biotin-labeled cRNA was hybridized onto Mouse- 6 v1 Expression BeadChips (Illumina Inc, San Diego, CA, USA), representing $>46,000$ probe sequences per array, for 16 hours at $55^{\circ} \mathrm{C}$. BeadChips were then scanned using Illumina's BeadStation 500X gene expression system (Illumina Inc., San Diego, CA). Fluorescence images of the Illumina BeadArrays were translated into relative expression levels using the Bioconductor [23] package beadarray [24] with standard parameter settings. The intensity values of the arrays were log2-transformed and quantile-normalized for each tissue separately.

\section{Analyses of expression data}

ANOVA was performed to evaluate differences between (i) the BFMI and B6 mice on SMD and (ii) between BFMI mice on SMD and HFD using the SAS 9.1 statistical software package (SAS Institute Inc., Cary, NC,
USA). For body composition and serum parameters, the Wilcoxon rank sum test was used and for the comparison of the adipocyte diameters, t-tests for two group comparisons were used. P-values smaller than 0.05 were considered statistically significant. For the comparison of time courses for weight and fat gain, longitudinal data were subjected to ANOVA with repeated measurement.

To measure the significance of differential expression two separate t-tests were performed for each oligo in each tissue: BFMI on SMD vs. B6 on SMD and BFMI on HFD vs. BFMI on SMD. To address multiple testing, local false discovery rates (local FDR) were calculated. For this purpose a customized algorithm following Aubert et al. [25] was implemented in R. Differentially expressed genes which had both p-values smaller than 0.05 and local FDRs smaller than 0.5 were considered statistically significant. Where necessary, medians of fold-changes and medians of significance values ( $p$-value and local FDR) were used to define unique values for each gene if multiple oligos were present on the chip that measured the expression of the same gene. Genes showing significant differences between groups were assigned to the PPAR pathway based on the Kyoto Encyclopedia of Genes and Genomes (KEGG) database [26]. Pathways were visualized with GenMAPP [27].

\section{Acknowledgement}

We thank Ulf Kiesling and Marion Bütow for mouse husbandry and Pamela Kepper for technical assistance. This research was supported by grants from the German National Genome Research Network (NGFN: 01GS0486, 01GS0829) and by a grant of the German Research Foundation (GRK1209). We thank Syeda Anum for carefully reading the manuscript.

\section{Author details}

${ }^{1}$ Humboldt-Universität zu Berlin, Department for Crop and Animal Sciences, Invalidenstraße 42, 10115 Berlin, Germany. ${ }^{2}$ Freie Universität Berlin, FB Veterinärmedizin, Institut für Tierpathologie, Robert-von-Ostertag-Straße 15, 14163 Berlin, Germany. ${ }^{3}$ Max-Planck-Institut für molekulare Genetik, Ihnestraße 73, 14195 Berlin, Germany.

\section{Authors' contribution}

AW planned the mouse experiments and drafted the manuscript. HFG performed the analyses of the microarray data. AOS participated in the analyses of the microarray data and the statistical analyses. SM prepared the histological staining. ADG participated in the histological studies. RR performed the chip analysis. GAB designed the BFMI model, coordinated the study and contributed to the manuscript. All authors read and approved the final manuscript.

\section{Competing interests}

The authors declare that they have no competing interests.

Received: 9 August 2010 Accepted: 10 September 2010 Published: 10 September 2010

\section{References}

1. Barak Y, Kim S: Genetic Manipulations of PPARs: Effects on Obesity and Metabolic Disease. PPAR Res 2007, 2007:12781.

2. Evans RM, Barish GD, Wang YX: PPARs and the complex journey to obesity. Nat Med 2004, 10(4):355-361. 
3. Kersten S: Peroxisome proliferator activated receptors and obesity. Eur $J$ Pharmacol 2002, 440(2-3):223-234.

4. Guerre-Millo M, Gervois P, Raspé E, Madsen L, Poulain P, Derudas B, Herbert JM, Winegar DA, Willson TM, Fruchart JC, Berge RK, Staels B: Peroxisome proliferator-activated receptor alpha activators improve insulin sensitivity and reduce adiposity. J Biol Chem 2000, 275:16638-16642.

5. Edvardsson U, Bergstrom M, Alexandersson M, Bamberg K, Ljung B, Dahllof B: Rosiglitazone (BRL49653), a PPAR-gamma-selective agonist, causes peroxisome proliferator-like liver effects in obese mice. $J$ Lipid Res 1999, 40(7):1177-1184.

6. Larsen PJ, Jensen PB, Sorensen RV, Larsen LK, Vrang N, Wulff EM, Wassermann K: Differential influences of peroxisome proliferatoractivated receptors gamma and -alpha on food intake and energy homeostasis. Diabetes 2003, 52(9):2249-2259.

7. Kadowaki T, Hara K, Kubota N, Tobe K, Terauchi Y, Yamauchi T, Eto K, Kadowaki H, Noda M, Hagura R, Akanuma Y: The role of Ppar- $\gamma$ in high-fat diet-induced obesity and insulin resistance. J Diabetes Complications 2002, 16(1):41-45

8. Rosen ED, Sarraf P, Troy AE, Bradwin G, Moore K, Milstone DS, Spiegelman BM, Mortensen RM: Peroxisome proliferator-activated receptor-gamma is required for the differentiation of adipose tissue in vivo and in vitro. Mol Cell 1999, 4:611-617.

9. Wagener A, Schmitt AO, Aksu S, Schlote W, Neuschl C, Brockmann GA: Genetic, sex, and diet effects on body weight and obesity in the Berlin Fat Mouse Inbred lines. Physiol Genomics 2006, 27:264-270.

10. Meyer CW, Wagener A, Rink N, Hantschel C, Heldmaier G, Klingenspor M, Brockmann GA: High energy assimilation and altered lipid metabolism are causes of obesity in the BFMI line. Obesity 2009, 17(11):1988-1993.

11. Neuschl C, Hantschel C, Wagener A, Schmitt AO, Illig T, Brockmann GA: A unique genetic defect on chromosome 3 is responsible for juvenile obesity in the Berlin Fat Mouse. Int J Obesity

12. Memon RA, Tecott LH, Nonogaki K, Beigneux A, Moser AH, Grunfeld C, Feingold KR: Up-regulation of peroxisome proliferator-activated receptors (PPAR-alpha) and PPAR-gamma messenger ribonucleic acid expression in the liver in murine obesity: troglitazone induces expression of PPARgamma-responsive adipose tissue-specific genes in the liver of obese diabetic mice. Endocrinol 2000, 141(11):4021-4031.

13. Knauf C, Rieusset J, Foretz M, Cani PD, Uldry M, Hosokawa M, Martinez E, Bringart M, Waget A, Kersten S, Desvergne B, Gremlich S, Wahli W, Seydoux J, Delzenne NM, Thorens B, Burcelin R: Peroxisome proliferator activated receptor-alpha-null mice have increased white adipose tissue glucose utilization, GLUT4, and fat mass: Role in liver and brain. Endocrinol 2006, 147(9):4067-4078.

14. Patsouris $D$, Reddy JK, Müller $M$, Kersten S: Peroxisome proliferatoractivated receptor-alpha mediates the effect of high-fat diet on hepatic gene expression. Endocrinol 2006, 147(3):1508-1516.

15. Harrity T, Farrelly D, Tieman A, Chu C, Kunselman L, Gu L, Ponticiello R, Cap M, Qu F, Shao C, Wang W, Zhang H, Fenderson W, Chen S, Devasthale P, Jeon Y, Seethala P, Yang WP, Ren J, Zhou M, Ryono D, Biller S, Mookhtiar KA, Wetterau J, Gregg R, Cheng PT, Hariharan N: Muraglitazar: a novel dual (alpha/gamma) peroxisome proliferatoractivated receptor activator, improves diabetes and other metabolic abnormalities and preserves beta-cell function in $d b / d b$ mice. Diabetes 2006, 55(1):240-248.

16. Way JM, Harrington WW, Brown KK, Gottschalk WK, Sundseth SS, Mansfield TA, Ramachandran RK, Willson TM, Kliewer SA: Comprehensive messenger ribonucleic acid profiling reveals that peroxisome proliferator-activated receptor gamma activation has coordinate effects on gene expression in multiple insulin-sensitive tissues. Endocrinology 2001, 142(3):1269-1277.

17. Boss $\mathrm{O}$, Muzzin $\mathrm{P}$, Giacobino JP: The uncoupling proteins, a review. Eur $J$ Endocrinol 1998, 139:1-9.

18. Hageman RS, Wagener A, Hantschel C, Svenson KL, Churchill GA, Brockmann GA: High fat diet leads to increased storage of monounsaturated fatty acids and tissue specific risk factors for diseases in DBA/2 mice. Physiol Genomics 2010, 42(1):55-66.

19. Kubota N, Terauchi Y, Miki H, Tamemoto H, Yamauchi T, Komeda K, Satoh S, Nakano R, Ishii C, Sugiyama T, Eto K, Tsubamoto Y, Okuno A, Murakami K, Sekihara H, Hasegawa G, Naito M, Toyoshima Y, Tanaka S, Shiota K, Kitamura T, Fujita T, Ezaki O, Aizawa S, Nagai R, Tobe K, Kimura S,
Kadowaki T: PPAR gamma mediates high-fat diet-induced adipocyte hypertrophy and insulin resistance. Mol Cell 1999, 4(4):597-609.

20. Wang YX, Lee CH, Tiep S, Yu RT, Ham J, Kang H, Evans RM: Peroxisome proliferator-activated receptor delta activates fat metabolism to prevent obesity. Cell 2003, 113(2):159-170.

21. Tinsley FC, Taicher GZ, and Heiman ML: Evaluation of a quantitative magnetic resonance method for mouse whole body composition analysis. Obes Res 2004, 12:150-160.

22. Einstein $\mathrm{FH}$, Atzmon $\mathrm{G}$, Yang XM, Ma XH, Rincon M, Rudin E, Muzumdar R, Barzilai N: Differential responses of visceral and subcutaneous fat depots to nutrients. Diabetes 2005, 54(3):672-678.

23. Reimers M, Carey VJ: Bioconductor: an open source framework for bioinformatics and computational biology. Meth Enzymol 2006, 411:119-134.

24. Dunning MJ, Smith ML, Ritchie ME, Tavare S: Beadarray: R classes and methods for Illumina bead-based data. Bioinformatics 2007, 23:2183-2184

25. Aubert J, Bar-Hen A, Daudin JJ, Robin S: Determination of the differentially expressed genes in microarray experiments using local FDR. BMC Bioinformatics 2004, 5:125.

26. Kyoto Encyclopedia of Genes and Genomes. [http://www.genome.jp/ $\mathrm{kegg} / \mathrm{J}$.

27. Dahlquist KD, Salomonis N, Vranizan K, Lawlor SC, Conklin BR: GenMAPP, a new tool for viewing and analyzing microarray data on biological pathways. Nat Genet 2002, 31(1):19-20.

doi:10.1186/1476-511X-9-99

Cite this article as: Wagener et al:: Genetic and diet effects on Ppar- $\alpha$ and Ppar- $\gamma$ signaling pathways in the Berlin Fat Mouse Inbred line with genetic predisposition for obesity. Lipids in Health and Disease 2010 9:99.

\section{Submit your next manuscript to BioMed Central and take full advantage of:}

- Convenient online submission

- Thorough peer review

- No space constraints or color figure charges

- Immediate publication on acceptance

- Inclusion in PubMed, CAS, Scopus and Google Scholar

- Research which is freely available for redistribution

Submit your manuscript at www.biomedcentral com/submit
C Biomed Central 